
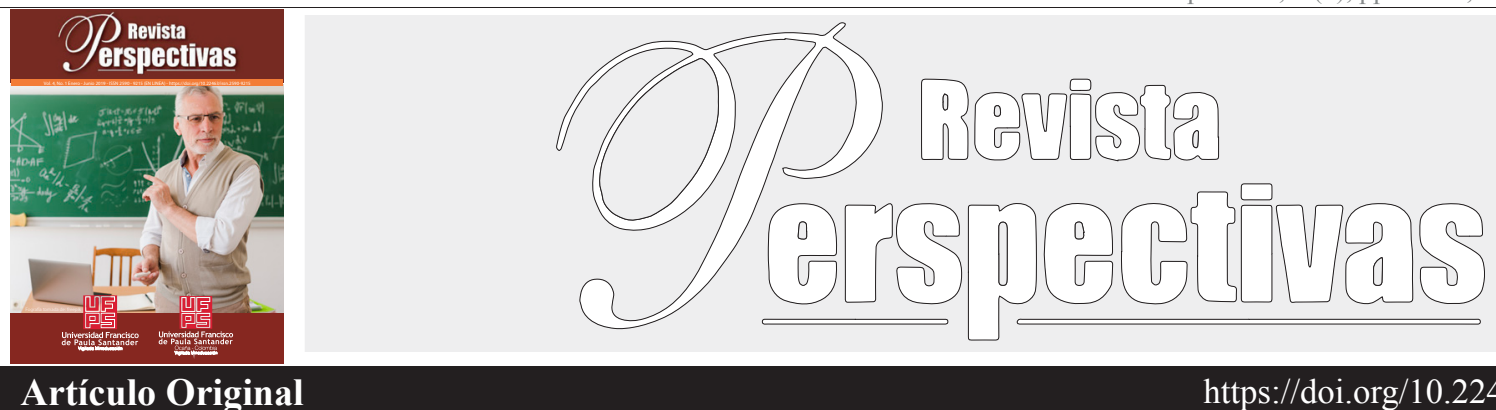

Artículo Original

https://doi.org/10.22463/25909215.1752

\title{
Actitudes hacia las Matemáticas y prácticas docentes: un estudio exploratorio en maestros
}

Attitudes towards Mathematics and teaching practices: an exploratory study with teachers

Atitudes em relação à Matemática e às práticas pedagógicas: um estudo exploratório em professores

Ariadna Gómezescobar-Camino ${ }^{\mathrm{a}}$, Carmen María León-Mantero ${ }^{\mathrm{b}}$, Raquel Fernández-Cezar ${ }^{\mathrm{c}^{*}}$

a Doctora en Investigación Educactiva, ariadna.gomez@uclm.es, ORCID 0000-0001-5104-6269, Universidad de Castilla-La Mancha, Toledo, España.

boctora en Ciencias Sociales y Jurídicas, cmleon@uco.es, ORCID 0000-0002-2870-8807, Universidad de Córdoba, Córdoba, España.

Doctora universidad Autonoma de madrid, raquel.fcezar@uclm.es, ORCID 0000-0002-9013-7734, Universidad de Castilla-La Mancha, Toledo, España.

Cómo citar: Gómezescobar, A., León, C. M., \& Fernández, R. (2019). Actitudes hacia las Matemáticas y prácticas docentes: un estudio exploratorio en maestros. Perspectivas, 4(1), 23-31.

Recibido: Agosto 20, 2018; Aceptado: Noviembre 01, 2018

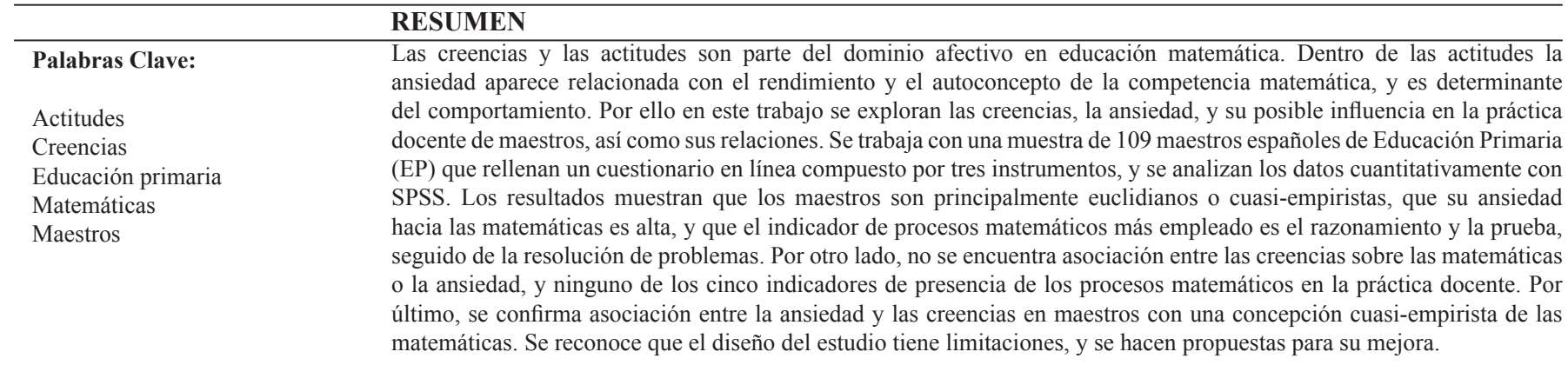

\section{ABSTRACT}

Keywords:

Beliefs and attitudes are part of the affective domain in mathematics education. Within the attitudes, anxiety appears in the literature as the most directly related to achievement and self-concept of competence in mathematics, and behavior determinant. Therefore, this work explores teachers' beliefs, anxiety and the presence of mathematical processes in the

Attitudes

Beliefs teaching practices, as well as the possible relationships among them. The sample used is of proximity consisting on 109

Primary education Primary Education Spanish mathematics teachers who complete an online questionnaire composed by three instruments, Mathematics and analyze the data quantitatively with the SPSS statistical package. The results show that the teachers exhibit mainly either euclidean or quasiempirical beliefs towards mathematics, that anxiety towards mathematics is high, and that the most used indicators of mathematical processes in teaching practice is reasoning and proof, followed by problem solving. On the other hand, there is no association between beliefs or anxiety towards mathematics, on the one side, and the five indicators of the presence of mathematical processes in teaching practice on the other. Finally, it is confirmed the association between anxiety and the quasi-empirical belief of mathematics. Limitations on the design of the experiment are identified, and future proposals to improve it are indicated.

\begin{tabular}{ll}
\hline RESUMO \\
\hline Palavras chave: & Crenças e atitudes fazem parte do domínio afetivo na educação matemática. Nas atitudes, a ansiedade aparece \\
& relacionada ao desempenho e autoconceito da competência matemática e é determinante do comportamento. Portanto, \\
& neste trabalho exploramos as crenças, a ansiedade e sua possível influência na prática docente dos professores, bem \\
Atitudes & como suas relações. Trabalhamos com uma amostra de 109 professores de Educação Primária (EP) espanhóis que \\
Crenças & preenchem um questionário online composto por três instrumentos e analisam os dados quantitativamente com \\
Ensino fundamental ${ }^{\circ}$ & o SPSS. Os resultados mostram que os professores são principalmente euclidianos ou quase empiristas, que sua \\
Matemática & ansiedade em relação à matemática é alta e que o indicador de processo matemático mais utilizado é o raciocínio e \\
Professores & o teste, seguidos pela resolução de problemas. Por outro lado, não há associação entre crenças sobre matemática ou \\
& ansiedade e nenhum dos cinco indicadores da presença de processos matemáticos na prática docente. Por fim, \\
& uma associação entre ansiedade e crenças em professores é confirmada por uma concepção quase empírica da \\
& matemática. Reconhece-se que o desenho do estudo tem limitações e propostas são feitas para o seu aprimoramento.
\end{tabular}

*Autor de Correspondencia.

E-mail: raquel.fcezar@uclm.es (Raquel Fernández Cezar)

(c) (i) $\Theta$ Peer review is the responsibility of the Universidad Francisco de Paula Santander.

This is an article under the license CC BY-ND 


\section{Introducción}

El reconocimiento de la importancia de los factores afectivos (emociones, creencias, actitudes y valores) en la enseñanza y el aprendizaje de las matemáticas (Goldin, 2002; Gómez-Chacón, 2000a; Hannula, Evans, Philippou y Zan, 2004) hace que sea necesario su estudio tanto en el alumnado como en los maestros de matemáticas. El término actitud se ha usado en Educación Matemática con una definición menos clara que en psicología, donde se ha considerado como algo observable y medible mediante instrumentos diseñados para medir distintas componentes de la misma (Mcleod, 1992). A lo largo del siglo XX todas las definiciones revisadas de actitud incluyen una componente comportamental, pues se la considera como una fuerza motivacional del comportamiento humano. Sin embargo, no existe consenso en su definición en el ámbito de la Educación Matemática (Di martino y Zan, 2003, 2011; Mcleod y Adams, 2012). En este trabajo tomamos la definición de actitud de Gómez-Chacón (2000b, p. 23): "Predisposición evaluativa de conducta que determina las intenciones personales e influye en el comportamiento". Por lo tanto, consiste en tres componentes: una cognitiva, que se manifiesta en las creencias respecto de la materia; otra afectiva, que se manifiesta en el trabajo o influye en los sentimientos de aceptación o rechazo, y otra intencional o tendente a un cierto comportamiento, que se manifiesta en la práctica docente en los maestros. Consideramos, por tanto, la actitud desde una perspectiva multidimensional. Los estudios que han tratado de establecer alguna vinculación entre las distintas componentes son muy diferentes y poco comparables, y además aportan resultados contradictorios: Ma y Kishor (1997) no encuentran relación significativa entre el rendimiento académico y la actitud. Sin embargo, en estudios más recientes (Hidalgo, Maroto, y Palacios, 2005; Estrada, 2007; Autor, 2010, 2016; Sánchez, Segovia Y Miñán, 2011; Mellado, Blanco, borrachero y Cárdenas, 2012; Blanco, Guerrero-Barona Y CaballeroCarrasco, 2013; Picos, Alonso, Saéz y Del rincón, 2013; Nortes y Nortes, 2014) se reconoce la vinculación del aspecto cognitivo y afectivo en estudiantes de grado de maestro. No obstante, los trabajos sobre el dominio afectivo realizados con maestros de Educación Primaria (Autores, 2016; Autor, 2018) son pocos, y también menos abundantes en el entorno iberoamericano que en el anglosajón. En este último contexto se encuentran diferentes trabajos ya desde finales del siglo pasado (Wood, 1988; Sayers, 2007; Thiel, 2010; Sweeting, 2011; Fives, Lacatena y Gerard, 2015), y más recientemente van apareciendo estudios también en otros ámbitos geográficos (Uysal y Dede, 2016).

Por la importancia del magisterio en las primeras edades, este trabajo se centra en el estudio de las actitudes hacia las matemáticas de los maestros los maestros de Educación Primaria y su posible relación con su práctica docente. Existen diversas consideraciones por las que es interesante saber lo que ocurre en este colectivo desde el punto de vista de la Educación
Matemática: primero por la escasez de estudios que tratan este tema en el ámbito español, y segundo por la posibilidad de que esta actitud influya sobre su práctica docente y el rendimiento de sus alumnos. Por ejemplo, algunos autores han señalado que las creencias y emociones hacia las matemáticas de los maestros influyen en el rendimiento, actitudes y creencias hacia esta disciplina de sus alumnos (Caballero, Blanco, y Guerrero, 2008; Ernest, 1989; Pezzia y di martino, 2011). Según Maaß y Schlöglmann (2009), las actitudes hacia las matemáticas son adquiridas a lo largo de la etapa de estudiante, lo que enfatiza el papel del maestro en las mismas. Además, otros autores señalan que el desarrollo de actitudes positivas hacia las matemáticas depende del estilo del maestro que se haya tenido (Rivera y Gómez-Chacón, 2013). Por lo tanto, las actitudes negativas pueden aparecer cuando no existe idoneidad didáctica (Vásquez, 2010), posiblemente debida a la falta de adecuación del estilo de enseñanza del maestro al de aprendizaje del alumno. Ocurre, por ejemplo, cuando el maestro enseña matemáticas de manera instrumental y el alumno las aprendería mejor de manera relacional (Amato, 2004). Por otro lado, otros investigadores aseguran que las variables afectivas, como las creencias y las actitudes hacia las matemáticas, son determinantes en el diseño de la praxis de aula de la materia (Frade Y Gómez-Chacón, 2009; Hodgen Y Askew, 2006; Philippou y Christou, 1998, 2002).

Por todo lo expuesto, los objetivos que nos planteamos en este trabajo son explorar la actitud de los maestros españoles de EP desde una concepción multidimensional, así como las posibles relaciones que pudieran determinarse entre las distintas dimensiones.

\section{Marco teórico}

Como ya se ha dicho anteriormente, a lo largo del siglo $\mathrm{XX}$ se han venido estudiando las actitudes en educación, y particularmente en Educación Matemática, sin que se haya encontrado una definición de consenso (Feirabend, citado en Aiken, 1970; Aiken, 1970; Hart, 1989). Aiken (1970) realiza una revisión de los trabajos de esa década y reconoce que aunque no se cuenta con una definición para actitud en Educación Matemática, hay un consenso en reconocer que tiene un aspecto conductual y comportamental. Respecto a los modelos dimensionales, no existe tampoco acuerdo. Un modelo de estudio sobre la actitud hacia las matemáticas lo constituye en el entorno iberoamericano el estudio de GairínSallán (1991), estudio en el cual no se detallan las dimensiones consideradas. Por otro lado, McLeod (1992) propone un modelo sobre el dominio afectivo en el aprendizaje matemático considerando que está compuesto por actitud, creencias y emociones. Otros autores como DeBellis y Goldin (1999) proponen un modelo en el que recogen 4 constructos: actitudes, creencias, emociones y valores. En este trabajo se considera un modelo de tres dimensiones (Gómez-Chacón, 2000a,b). 


\section{Actitudes hacia las matemáticas}

Auzmendi (1992) considera distintas componentes dentro de la actitud hacia las matemáticas: utilidad, agrado, motivación, confianza y ansiedad. De todas ellas, la ansiedad es la que se considera en la bibliografía más directamente relacionada con el rendimiento y el autoconcepto de la competencia en matemáticas (Picos, Alonso, Saez y Del rincón, 2013). Etimológicamente la palabra ansiedad, del latín "anxietas", significa angosto, estrecho, porque esta emoción se manifiesta con un sentimiento de opresión, o sensación de estar apretado. Lang (citado en Carpintero, 2000) acuñó el término de "triple sistema de respuesta", según el cual la ansiedad se manifiesta en los sistemas de respuesta cognitivo, fisiológico y motor. La respuesta cognitiva hace referencia a pensamientos de anticipación, de valoración de los recursos personales, atención, concentración. El sistema de respuesta fisiológica se refiere a la activación del sistema nervioso autónomo, y la respuesta motora, se manifiesta mediante la inquietud o la evitación de las situaciones percibidas como causantes de ansiedad (Carpintero, 2000). Si la persona percibe las matemáticas como amenazantes, los niveles de ansiedad pueden bloquear e incluso hacerle que evite afrontar situaciones asociadas con ellas. Aunque el sentimiento de ansiedad presenta otra vertiente, siendo necesaria en niveles adecuados, pues permite a la persona afrontar las tareas de esta asignatura con eficiencia.

Las matemáticas, pues, pueden constituir situaciones amenazantes o no dependiendo de la interpretación de cada persona: en el caso del alumno, pensar que van a mostrar su (supuesta) limitada capacidad y torpeza; o en el caso del docente, creer que su alumnado percibirá sus limitaciones como profesor (Dogan, 2012).

\section{Creencias sobre las matemáticas}

Algunos autores han indagado sobre esta componente de la actitud (Baroody y Coslick, 1998; Goldin, Rösken y Törner, 2009), no encontrándose consenso ni sobre su definición ni sobre un modelo que sea internacionalmente aceptado por los investigadores en Educación Matemática. Algunos autores defienden la importancia de dichos modelos, y otros, contribuyen con definiciones para caracterizarlas como parte del dominio afectivo, como por ejemplo Gómez-Chacón (2003).

En este sentido, los estudios acerca de las creencias sobre las Matemáticas de los docentes, se desarrollan en dos líneas principales de investigación (Hannula et al., 2016): la relación entre las creencias y la práctica docente; y los cambios en las creencias de los mismos. Con respecto a la primera de ellas, esta reciente revisión del estado de la cuestión, pone de manifiesto que no hay datos concluyentes, pues en algunos casos son inconsistentes las relaciones encontradas, observándose también que los factores que pueden influir en las creencias, como el conocimiento, la experiencia, los objetivos o el contexto de los docentes, deben ser estudiados en profundidad.

Existen estudios que abordan las creencias de los docentes sobre algún dominio concreto de las Matemáticas, como la Geometría (Pérez y Guillén, 2007). Sin embargo, existen pocas publicaciones que centren su interés en las creencias sobre las Matemáticas en maestros de las primeras etapas de la educación (Vásquez, 2010; Donoso, 2015; Martín, 1998).

\section{Prácticas docentes}

En las investigaciones sobre la práctica de aula, encontramos algunos trabajos que analizan aspectos generales no específicamente relacionados con la Educación Matemática (Ross, Mcdougall, Hogaboam-Gray y Lesage, 2003); otros realizados con el objeto de elaborar un modelo sobre dicha praxis particularmente en Educación Matemática (Godino, contreras y Font, 2006; Font, Planas y Godino, 2010). Otros trabajos se centran en mostrar casos que sugieren indicaciones sobre lo que serían buenas prácticas de aula para ayudar al profesorado de distintas etapas educativas a abordar y desarrollar las mismas (Planas y Alsina, 2009). Sin embargo, son escasos los estudios que reportan evidencias sobre las prácticas de enseñanza de matemáticas en Educación Infantil y Primaria. Se aportan estas en el trabajo de Vásquez (2010), que analiza la concepción de las maestras de preescolar sobre la enseñanza, el aprendizaje y la evaluación en matemáticas, y concluye que tiene una amplia presencia la enseñanza memorística por exposición, entendiendo los docentes que con ella consiguen aprendizaje al lograr que el niño repita. Sin embargo, esta enseñanza expositiva de la Matemática no es considerada efectiva, pues el aprendizaje que consigue es memorístico, y éste no es considerado significativo por el autor. El docente no lo es solo porque transmite conocimiento, sino también porque gestiona adecuadamente la clase. Existen algunos modelos que caracterizan el conocimiento profesional del docente de matemáticas (Hill, Ball y Schilling, 2008; Godino, 2009). Y respecto a la práctica de aula, los autores aconsejan darle prioridad al razonamiento y a la resolución de problemas (Villella, 2002).

En el diseño de esa práctica de aula, los docentes se apoyan en los documentos o currículos oficiales. Estos se han ido desarrollando durante el pasado siglo en distintos países, entre ellos España. Consultando en concreto los currículos de matemáticas más recientes (Real Decreto 126/2014 [rd 126/2014] (2014) para ed. primaria , y Real Decreto 1105/2014 [rd 1105/2014] (2014) para eso y bachillerato) se observa que están orientados fundamentalmente a la adquisición de conocimientos. A pesar del cambio producido en las leyes europeas hacia las competencias entendidas como el "saber hacer", lo que recoge la última ley española, la LOMCE (RD 126/2014 (2014) y RD 1105/2014 (2014) son los estándares 
evaluables, formulados como indicadores de adquisición de contenidos. En ambos reales decretos hay escasas referencias a la práctica docente recomendada, hablando explícitamente solo de la resolución de problemas.

Sin embargo, en EEUU el National Council of Teachers of Mathematics (NCTM, 2003) recogió en un documento los que considera como los procesos fundamentales en la Educación Matemática, reconociendo, además de la resolución de problemas, razonamiento y prueba, conexiones de la matemática con otras áreas, representación de las ideas y procesos matemáticos, y comunicación de argumentos matemáticos. Nos parece adecuado tomarlos como referencia para caracterizar la práctica docente de los maestros en Educación Matemática. En el ámbito de habla hispana, Alsina y Coronata (2014) han analizado la presencia de los procesos matemáticos señalados por el NCTM en la práctica docente de maestros de Educación Infantil. Tomaremos como referencia este trabajo por la cercanía con nuestro contexto geográfico y sociocultural.

Por todo lo expuesto, este trabajo pretende analizar la actitud hacia las matemáticas de los maestros de Educación primaria (EP) en España y los factores que pudieran influir en ellas (el sexo, los años de experiencia, el tipo de centro en el que trabajan los maestros y el tipo de formación universitaria recibida). También se explorarán las posibles relaciones entre las dimensiones de dicha actitud.

\section{Materiales y métodos}

Se emplea una metodología cuantitativa y se aportan estadísticos descriptivos y correlacionales de las dimensiones o variables estudiadas.

\section{Muestra}

El muestreo es de proximidad, y la muestra de conveniencia, pues la conforman los maestros de EP que han accedido a contestar el cuestionario en línea. La componen 109 maestros en ejercicio en España (69 mujeres), mayoritariamente trabajadores del sistema público de educación (87), y con más de 20 años de experiencia (71), provenientes principalmente de los programas de formación de maestros mediante diplomatura (52) o que han completado una posterior licenciatura (39).

\section{Instrumento}

Lo constituye un cuestionario en línea que incluye las tres dimensiones de la actitud consideradas: las creencias sobre las matemáticas, la ansiedad, y la presencia de los procesos matemáticos en la práctica docente. Para las creencias se emplea el cuestionario de creencias de Baroody y Coslick (1998), para la ansiedad se emplea el cuestionario de Auzmendi (1992) modificado (Autores, 2016); y para la presencia de los procesos matemáticos en la práctica docente se emplea el cuestionario de Alsina y Coronata (2014). Todos están validados por sus autores en las referencias que se indican.

\section{Análisis estadístico}

Se emplea SPSS (Versión 24.0; IBM Corp., 2016). Las respuestas a los cuestionarios se plantean mediante escala Likert de 1 a 5 donde 1 es totalmente en desacuerdo y 5 es totalmente de acuerdo. Se toman todas como variables métricas, dado que según Díaz (2002) una variable ordinal con 5 categorías o más puede considerarse como métrica.

Se analiza en primer lugar la consistencia interna y validez de los instrumentos empleados mediante alfa de Cronbach. Si esta no tiene un valor razonablemente alto para ciencias sociales, se amplía el estudio de adecuación del instrumento mediante análisis factorial exploratorio.

Para la cuantificación de la ansiedad se suman los 10 ítems del cuestionario, y al total se llama ANS. Se toman en sentido positivo, por lo que, para mantener la coherencia del análisis, los ítems 2.5 y 2.7 se invierten.

El cuestionario de creencias tiene 9 ítems distribuidos en tres bloques: 1.1-1.3, relativos a una concepción Euclidiana Platónica de las matemáticas, a cuyo total se llama E; 1.4-1.6, a una concepción cuasi empirista, a cuyo total se llama CE; y 1.71.9 a una concepción constructivista, a cuyo total se llama C. Los ítems que están formulados de manera negativa son invertidos para mantener la coherencia del análisis. Consecuentemente, los ítems del bloque C-E se invierten. Para incluir a cada maestro en una, su puntuación en los totales de cada grupo de ítems, E, CE y C, debe estar entre 9 y 15. Además, dado que la mayoría de los maestros no muestra una concepción pura, puntuando en las tres categorías, en este trabajo se les asigna a aquella categoría en la que puntúen más alto.

Para analizar la presencia de los procesos en la práctica docente empleamos el análisis que propone el autor (Alsina y Coronata, 2014) enfocado a determinar la suficiencia de la presencia de cada uno de los procesos en la práctica docente. Los indicadores de la presencia de los procesos matemáticos son: resolución de problemas, razonamiento y prueba, conexiones con otras áreas, comunicación, y representaciones. Serán llamados en adelante como sigue: RP al indicador de presencia de resolución de problemas; al de razonamiento y prueba, RzP; al de conexiones, CON; al de comunicación, COM; al de representaciones, REP. Al emplear los umbrales sugeridos por el autor, las variables se convierten en dicotómicas tomando valor 1 cuando está presente y 0 cuando no lo está.

\section{Resultados y discusiones}


La media, desviación típica (DT) y otros estadísticos descriptivos de la ansiedad hacia las matemáticas se muestran en la tabla 1.

Tabla 1. Valores descriptivos de la ansiedad hacia las matemáticas (ans) en maestros españoles $(n=109)$

\begin{tabular}{cccccc}
\hline Variable & Rango & Mínimo & Máximo & Media & DT \\
\hline ANS & 28 & 22 & 50 & 39.06 & 7.07 \\
\hline
\end{tabular}

La consistencia interna de los ítems empleados se mide mediante alfa de Cronbach obteniendo un valor de .77, muy aceptable para ciencias sociales. La ansiedad media es 39.06, claramente por encima del valor neutro nominal (30), pero por debajo de la mediana que es 41 . Al valor neutro le corresponde un porcentaje acumulado de 14.3; el primer cuartil está en 34.5 , y el tercer cuartil en 45 . Por lo tanto, podemos decir que solo el $14.3 \%$ de los maestros presentan valores de ansiedad baja, considerando así a los valores inferiores al valor nominal neutro. Solo el $25 \%$ están por debajo de 34.5 , mientras que el $50 \%$ de los maestros muestra una ansiedad alta, entre 41 y 50. Este estudio está en la línea de lo observado por Wood (1988) cuando analiza la ansiedad hacia las matemáticas en maestros en ejercicio en Estados Unidos, y con el estudio más reciente realizado con maestros españoles de Autor (2018). Sin embargo, otros estudios realizados con los estudiantes del grado de maestro contrastan con estos resultados (AUTOR, 2010; PICOS ET AL. 2013), pues en ellos la ansiedad observada es baja. Sería interesante estudiar si esta diferente ansiedad observada entre los maestros en formación, y los maestros en ejercicio con años de experiencia es debida a factores relacionados con su percepción de las matemáticas en sí mismas, o con otros factores, como la diferencia en los programas de formación de maestros que se han sucedido en los últimos años, o los relacionados con su práctica docente.

En la tabla 2 se muestran los estadísticos descriptivos rango, valor mínimo, máximo, media y desviación típica de las creencias de los maestros de la muestra estudiada. Estos se distribuyen en las categorías de las creencias, E, C-E, C, según se indica en el análisis estadístico.

Tabla 2. Valores descriptivos de las creencias sobre las matemáticas en cada categoría $(n=109)$

\begin{tabular}{cccccc}
\hline Variable & Rango & Mínimo & Máximo & Media & DT \\
\hline $\mathrm{E}$ & 12 & 3 & 15 & 18.87 & 2.40 \\
$\mathrm{C}-\mathrm{E}$ & 10 & 5 & 15 & 10.85 & 2.57 \\
$\mathrm{C}$ & 7 & 6 & 13 & 9.52 & 1.75 \\
\hline
\end{tabular}

Se analiza la consistencia interna o fiabilidad mediante alfa de Cronbach obteniéndose .328 (.342 con elementos estandarizados) que es un valor bajo, incluso para estudios de ciencias sociales. Realizamos un análisis factorial exploratorio con método de extracción de máxima verosimilitud y rotación varimax con normalización de Kaiser, que ofrece un modelo de 3 factores con un coeficiente KMO y Bartlett de 0.618 $(p<.000)$. Este se incluye en la tabla 3, donde se observa que los ítems 1.1-1.3 presentan cargas superiores a .47 al factor 2 , los ítems $1.4 *-1.6 *$ presentan cargas superiores a .45 al factor 1 , mientras que los ítems 1.8 presenta una carga baja, $.339 \mathrm{al}$ factor 3 , el ítem 1.9 de .501 , y el ítem 1.7 presenta cargas muy bajas a todos los factores.

Tabla 3. Análisis factorial exploratorio del cuestionario de creencias sobre las matemáticas convergentes a 3 factores

\begin{tabular}{cccc}
\hline Item* & \multicolumn{3}{c}{ Factor } \\
& $\mathbf{1}$ & $\mathbf{2}$ & $\mathbf{3}$ \\
\hline 1.1 & -.191 & .471 & .088 \\
1.2 & .071 & .582 & .008 \\
1.3 & -.107 & .684 & .082 \\
$1.4^{*}$ & .998 & -.053 & .013 \\
$1.5^{*}$ & .718 & -.135 & -.154 \\
$1.6^{*}$ & .453 & -.170 & -.366 \\
1.7 & .192 & .121 & -.210 \\
1.8 & -.141 & .003 & .339 \\
1.9 & .089 & .210 & .501 \\
\hline
\end{tabular}

Se consideraría entonces que los ítems 1.1-1.3 (factor 2) nos darían información sobre maestros de creencias euclidianas, en la categoría E, y los ítems $1.4^{*}-1.6^{*}$ (factor 1) sobre la categoría cuasiemprirista, categoría C-E. Sin embargo, solo dos ítems, el 1.8 y el 1.9 podrían considerarse como informativos sobre la categoría constructivista, categoría C. Por otro lado, analizando el porcentaje acumulado de la varianza, un factor explica el $20.38 \%$ de la varianza, dos el $33.01 \%$, y tres el $39.48 \%$, siendo este muy bajo comparado con lo que sería exigible al modelo (aproximadamente un $60 \%$ ). Esta poca adecuación se refleja también en la prueba de bondad del ajuste en la que se obtiene $\mathrm{p}=.633$ (contraste $\chi 2(N=109 ; g l=12)=9.810)$. Por lo tanto, afianzamos la información sobre la fiabilidad de las categorías consideradas con cierta precaución.

En las creencias, cuanto más cercanos a 15 están los valores, más acorde es la concepción de las matemáticas que tiene el maestro con las características de sendas categorías. En la mayoría de los casos no se encuentran maestros con una concepción pura de uno de los tres tipos, sino que tienen visiones parciales que les hacen puntuar en los tres. Partiendo de esta situación, y dado que en este trabajo la asignación de cada maestro a una u otra categoría se realiza como se indicó en el apartado de análisis estadístico, el número de maestros en categoría E es 57 , en la categoría C-E se encuentran 43 , y en la categoría $\mathrm{C}, 5$, quedando 4 fuera de ellas. Estos números nos indican que los maestros españoles con una concepción constructivista de las matemáticas son una minoría (4.6\%). 
siendo más mayoritarios los grupos de maestros de concepción euclidiana o cuasiempirista ( $52.3 \%$ y $39.4 \%$, respectivamente).

Se ha explorado si alguno de los descriptores de la muestra (sexo, experiencia laboral, formación universitaria recibida, tipo de centro en el que trabaja) de manera individual podría influir en las creencias, encontrando que no lo hace el sexo $\left(\chi^{2}(\mathrm{~N}=109 ; \mathrm{gl}=3)=4.554, p=.208\right)$, el tipo de formación universitaria recibida $\left(\chi^{2}(\mathrm{~N}=109 ; \mathrm{gl}=3)=2.410, p=.983\right)$, el tipo de centro en el que ejerce su docencia $\left(\chi^{2}(\mathrm{~N}=109 ; \mathrm{gl}=6)\right.$ $=6.232, p=.398)$, mientras que marginalmente sí influyen en dichas creencias los años de experiencia $\left(\chi^{2}(\mathrm{~N}=109 ; \mathrm{gl}=15)=\right.$ $24.786, p=.053)$. Por lo tanto, no se espera que el desarrollo de un modelo de regresión multinomial para las creencias, considerando estos 4 factores como predictores, sea adecuado. Esta afirmación está apoyada por los resultados del test de bondad de ajuste del modelo mediante criterios de ajuste de logaritmo de la verosimilitud-2 $\left(\chi^{2}(\mathrm{~N}=109 ; \mathrm{gl}=33)=41.02\right.$, $p=.159$ ).

Retomando la dependencia marginalmente significativa entre las creencias y los años de experiencia, hay que destacar que los maestros con más años de experiencia manifiestan creencias euclidianas sobre las matemáticas, seguidos de los que muestran creencias cuasiempiristas.

En cuanto a la relación entre las distintas categorías de creencias se obtiene correlación negativa entre $\mathrm{C}$ y C-E, $\mathrm{R}_{\text {Pearson }}=-.248(\mathrm{p}=.018)$, lo que implicaría que cuanto más euclidiana es la visión que un maestro tiene de las matemáticas, menos cuasiempirista es dicha visión. Nos parece un resultado esperable, pues ambas concepciones de las matemáticas son parcialmente excluyentes, como proponen Baroody y Coslick (1998). Respecto a la correlación entre ansiedad y creencias, se encuentra una correlación significativa entre la categoría C-E y ANS $\left(\mathrm{R}_{\text {Pearson }}=-.248, \mathrm{p}=.023\right)$ lo que supondría que los maestros de creencias cuasiempiristas muestran menor ansiedad frente a las matemáticas. Esto podría significar que la concepción cuasi-empirista implica una concepción de las matemáticas más cercana a las ciencias experimentales. Los maestros con esta concepción de las matemáticas perciben la utilidad directa e inmediata de las mismas en la interpretación del mundo que les rodea, por lo que no muestran ansiedad en su uso para la interpretación de lo que ocurre a nuestro alrededor.

Los procesos matemáticos en la práctica docente considerados son 5. Dado que en la referencia del autor (ALSINA Y CORONATA, 2014) se proponen para maestros de Educación Infantil y en nuestro estudio los docentes son maestros de Educación Primaria, diferente etapa educativa, se considera pertinente estudiar la consistencia interna de la herramienta para nuestra muestra. Se estudia esta mediante el alfa de Cronbach, encontrándose el menor valor para el proceso comunicación, .769, y el mayor para el proceso representación, .835. Todos ellos se encuentran en valores muy aceptables en estudios del ámbito de las ciencias sociales.
Se analiza ahora la presencia de cada uno de los procesos en las prácticas docentes de los maestros y se muestran las frecuencias en la tabla 4.

Tabla 4. Tabla de frecuencias de uso de los procesos matemáticos en la práctica docente

\begin{tabular}{cc}
\hline Proceso matemático & Frecuencia \\
\hline Razonamiento y prueba $=\mathrm{RzP}$ & 102 \\
Conexiones $=\mathrm{COM}$ & 21 \\
Resolución de problemas $=\mathrm{RP}$ & 97 \\
Comunicación $=\mathrm{COM}$ & 55 \\
Representación $=\mathrm{REP}$ & 84 \\
\hline
\end{tabular}

El grupo más numeroso es el de maestros que emplean razonamiento y prueba, que casi asciende a la totalidad de la muestra, mientras que el menos numeroso es el que emplea conexiones, en menos de un quinto de la misma. Entre ellos se encuentran los que emplean resolución de problemas, casi la centena, mientras que el de comunicación lo emplea algo más de la mitad, y la representación es empleada por casi los cuatro quintos de la misma. Al analizar la posible relación entre la presencia de los procesos matemáticos en las prácticas docentes con la ansiedad y las creencias de los maestros, encontramos que solo el indicador RzP se asocia con las creencias sobre las matemáticas de los maestros en la categoría $\mathrm{E}$, creencias euclidianas $\left(\mathrm{R}_{\text {Spearmann }}=.193, p=.048\right)$, y ninguno de los procesos se asocia con la ANS $(p=.738)$.

La relación significativa y positiva, aunque con intensidad débil, entre el indicador razonamiento y prueba, y la categoría $\mathrm{E}$, podría interpretarse aduciendo que los maestros con concepción Euclidiana de las matemáticas las consideran compuestas y fundamentadas en argumentos lógicos, inductivos y deductivos, y que por lo tanto enfatizaría la presencia de la argumentación en su práctica docente, manifestada en la presencia del proceso de Razonamiento y prueba.

\section{Conclusiones}

En este trabajo se pretendía explorar las creencias, ansiedad hacia las matemáticas y prácticas docentes de maestros españoles de EP, así como las posibles relaciones que pudieran encontrarse entre estas variables. La ansiedad hacia las matemáticas en esta muestra presenta valores altos, en línea con otros estudios recientes realizados en España, y con lo encontrado en otros países. A nivel descriptivo, en las creencias se encuentra que los maestros que muestran una concepción constructivista de las matemáticas son una minoría, y que el grueso tiene una concepción euclidiana o cuasi-empirista casi a partes iguales. De los factores de la muestra analizados, sexo, formación universitaria, tipo de centro en el que trabajan y años de experiencia, solo este último tiene una influencia marginal sobre las creencias. 
En cuanto a la relación entre creencias y ansiedad hacia las matemáticas, encontramos que la ansiedad se relaciona con la concepción cuasi-empirista de las matemáticas, emergiendo una correlación negativa entre ellas. Por lo tanto, los maestros cuya concepción de las matemáticas es mayormente cuasi-empirista, muestran una menor ansiedad frente a las mismas. Respecto de la presencia de los procesos matemáticos en las prácticas docentes de los maestros, razonamiento y prueba, y resolución de problemas, aparecen como los más usados, en primer y segundo lugar, respectivamente. Conexiones aparece como el menos usado. Parece evidenciarse que la enseñanza de las matemáticas no se plantea estableciendo conexiones con otras áreas, luego los contextos de enseñanza interdisciplinares, si se emplean, no involucran a las matemáticas mayoritariamente. En cuanto a la existencia de relación entre la ansiedad hacia las matemáticas de los maestros, sus creencias sobre las mismas, y alguno de los procesos matemáticos presentes en la práctica docente, solo las respuestas de los maestros de concepciones mayormente euclidianas se asocian positiva, pero débilmente con el proceso de razonamiento y prueba, y con ninguno otro. Por tanto, no podemos decir de manera general que la concepción que los maestros tienen sobre las matemáticas determine la inclusión o exclusión de estos procesos matemáticos en su práctica docente. Respecto a la ansiedad, no se prueba que la ansiedad hacia las matemáticas influya en la presencia en la práctica docente de ninguno de los procesos matemáticos estudiados.

Este trabajo tiene claras limitaciones pues, en primer lugar, la muestra no es representativa, lo cual hace que las conclusiones no sean generalizables al territorio español; en segundo, la información se obtiene de manera autoinformada en el cuestionario sin contrastar con la visión de personas ajenas al propio sujeto de estudio: los maestros; y en tercero, la fiabilidad del cuestionario de creencias es excesivamente baja. La prospectiva de futuro sería mejorar el instrumento con el que se valoran las creencias hacia las matemáticas, elegir una muestra representativa de la población de maestros españoles, o, al menos, ampliarla para confirmar o no los resultados encontrados. Igualmente podría valorarse si tiene alguna influencia que la distribución del cuestionario se haga en línea, así como completar el estudio realizando entrevistas semiestructuradas a un grupo de trabajo elegido, para completar la instantánea del estado de este asunto contrabalanceando la autoinformación de los cuestionarios.

Implicaciones en los programas de formación de maestros. Las acciones deberían dirigirse a una enseñanza más constructivista y significativa de las matemáticas. Esta parece basada principalmente en la resolución de problemas y el razonamiento y prueba, pero no en el establecimiento de conexiones con otras áreas curriculares. Se propone que en los programas de formación de maestros exista espacio para el establecimiento de esas conexiones vía coordinación entre el profesorado universitario de diferentes áreas y asignaturas.
Particularmente importantes para el establecimiento de estas conexiones son las asignaturas o partes de las mismas que tratan el desarrollo curricular. A enfatizar las conexiones puede contribuir la adopción de una práctica docente universitaria en los programas de formación de maestros basada en la coordinación entre áreas, el planteamiento de trabajos en las asignaturas que requieran el diseño de tareas integradas con otras asignaturas. Sería una buena manera de aplicar el "aprender haciendo".

\section{Agradecimientos}

Este trabajo ha sido posible gracias al apoyo prestado por J. Carlos Toscano, del foro IBERCIENCIA de la Organización de Estados Iberoamericanos.

\section{Referencias}

Aiken, JR., \& Lewis, R. (octubre, 1970). Attitudes toward mathematics. Review of educational research, 40(4), 551-596.

Alsina, Áy Coronata, C. (junio, 2014). Los procesos matemáticos en las prácticas docentes: diseño, construcción y validación de un instrumento de evaluación. Edma 0-6: Educación Matemática en la Infancia ,3(2), 23-36.

Amato, S. A. (2004). Improving student teacher's attitudes to mathematics. En M.J. Hoines \& A.B. Fuglestad (Eds.), Proceedings of the 28th PME International Conference. Congreso llevado a cabo en Bergen, Noruega.

Auzmendi, E. (1992). Las actitudes hacia la matemáticaestadística en las enseñanzas media y universitaria. Características y medición. Bilbao: Mensajero.

Baroody, A y Coslick, R. T. (1998). Fostering children's mathematical power: An investigative approach to K-8 mathematics instruction. New Jersey: Lawrence Erlbaum Associates.

Blanco, L. J., Guerrero-Barona, E y Caballero-Carrasco, A. (septiembre, 2013). Cognition and affect in mathematics problem solving with prospective teachers. The Mathematics Enthusiast, 10(1), 335-363.

Caballero, A., Blanco, L. J y Guerrero, E. (junio, 2008). El dominio afectivo en futuros maestros de matemáticas en la universidad de Extremadura. Paradígma, 29(2), 157171 .

Carpintero, H. (agosto , 2000). Notas históricas sobre la ansiedad. Ansiedad y Estrés, 6(1), 1-19.

Debellis, V. A \& Goldin, G. A. (1999). Aspects of affect: Mathematical intimacy, mathematical integrity. En O. Zalavsky (Ed.), Proceedings of the 23rd conference of the International Group for the Psychology of Mathematics Education. Congreso llevado a cabo en Haifa, Isael.

Di Martino, P., \& Zan, R. (2003). What Does -Positivell Attitude Really Mean?. En N. Pateman., B. Dougherty \& 
J. Zillox (Eds.), Proceedings of the 2003 Joint Meeting of PME and PME-NA. Congreso lleado a cabo en Honolulu, Hawaii.

Di Martino, P., \& Zan, R. (diciembre, 2011). Attitude towards mathematics: a bridge between beliefs and emotions. ZDM, 43 (4), 471-482.

Díaz, V. (2002). Técnicas de Análisis Multivariante para Investigación Social y Comercial. Madrid: Ra-Ma.

Dogan, H. (febrero, 2012). Emotion, confidence, perception and expectation. Case of mathematics. International Journal of Science and Mathematics Education, 10(1), 49-69.

Donoso, P. M. (2015). Estudio de las concepciones y creencias de los profesores de educación primaria chilenos sobre la competencia matemática (Tesis doctoral, Universidad de Granada) .Recuperada de

h t t p : / / d i g i b u g. u g r. e s / b i t s t r e a m / handle/10481/42049/25633089.pdf;sequence=1

Ernest, P. (junio, 1989). The knowledge, beliefs and attitudes of the mathematics teacher: A model. Journal of education for teaching, 15(1), 13-33.

Estrada, A. (2007). Actitudes hacia la Estadística: un estudio con profesores de educación primaria en formación y en ejercicio. En M. Camacho., P. Flores y P. Bolea (Eds.), Actas del XI Simposio de la SEIEM. Congreso llevado a cabo en Santa Cruz de Tenerife, España.

AUTOR, 2010.

AUTORES, 2016.

AUTOR, 2018

Fives, H., Lacatena, N y Gerard, L. (diciembre, 2015). Teachers' beliefs about teaching (and learning). International handbook of research on teachers' beliefs, 62(3), 249265.

Font, V., Planas, N y Godino, J. D. (febrero, 2010). Modelo para el análisis didáctico en educación matemática. Infancia y Aprendizaje, 33(1), 89-105.

Frade, C y Gómez-Chacón, I. M. (2009). Researching Identity and Affect in Mathematics Education. En M. Tzekaki., M. Kaldrimidou y C. Sakonidis (Eds.), Procs. of the 33rd. Conference of the IGPME. Congreso llevado a cabo en Thessaloniki, Greece.

Gairín-Sallán, J. (1991). Las actitudes en educación: Un estudio sobre educación matemática. Barcelona: Marcombo.

Godino, J. D. (diciembre, 2009). Categorías de análisis de los conocimientos del profesor de matemáticas. UNION, Revista Iberoamericana de Educación Matemática, 20(1), 13-31.

Godino, J. D., Contreras, Á y Font, V. (septiembre, 2006). Análisis de procesos de instrucción basado en el enfoque ontológico-semiótico de la cognición matemática. Recherches en Didactique des Mathématiques, 26 (76), 39-88.

Goldin, G. (2002). Affect, Meta-Affect, and Mathematical Belief Structures. En G. Leder., E. Pehkonen \& G.
Törner (Eds.), Beliefs: A Hidden Variable in Mathematics Education? (pp. 59-73). Dordrecht: Kluwer Academic Publishers,

Goldin, G., Rösken, B \& Törner, G. (2009). Beliefs - no longer a hidden variable in mathematical teaching and learning processes. En J. Maaß \& W. Schloglmann (Eds.): Beliefs and attitudes in mathematics education: New research results (pp. 9-28). Rotterdam: Sense Publishers.

Gómez-Chacón, I. M. (septiembre, 2000a). Affective influences in the knowledge of mathematics. Educational Studies in Mathematics, 43(2), 149-168.

Gómez-Chacón, I. M. (2000b). Matemática emocional: Los afectos en el aprendizaje matemático. Madrid: Narcea.

Gómez-Chacón, I. M. (febrero, 2003). La tarea intelectual en matemáticas afecto, meta-afecto y los sistemas de creencias. Boletín de la Asociación Matemática Venezolana, 10(2), 225-247.

Hannula, M. S., Di Martino, P., PANTZIARA, M., Zhang, Q., Morselli, F., Heyd-Metzuyanim, E \& Goldin, G. (2006). Attitudes, Beliefs, Motivation, and Identity in Mathematics Education. En G.A. Goldin., M.S. Hannula., E. Heyd-metzuyanim., A. Jansen., R. Kaasila., S. Lutovac., P. Di Martino., F. Morselli., J.A. Middleton., M. Pantziara y Q. Zhang (Eds.), Attitudes, Beliefs, Motivation and Identity in Mathematics Education, (pp. 1-359. Cham: Springer,

Hannula, M., Evans, J., Philippou, G. N., \& ZAN, R. (coordinators) (JUNIO, 2004). Affect in Mathematics Education-Exploring Theoretical Frameworks. International Group fo the Psychology of Mathematics Education, 1(1), 107-136.

Hart, L. E. (1989). Describing the affective domain: Saying what we mean. En D.B. Mcleod y V.M. Adams (Eds.), Affect and mathematical problem solving, (pp. 37-45). New York: Springer.

Hidalgo, S., Maroto, A y Palacios, A. (agosto, 2005) . El perfil emocional matemático como predictor de rechazo escolar: relación con las destrezas y los conocimientos desde una perspectiva evolutiva. Educación Matemática, 17(2), 89-116.

Hill, H. C., Ball, D. L., \& Schilling, S. G. (febrero, 2008). Unpacking pedagogical content knowledge: Conceptualizing and measuring teachers' topicspecific knowledge of students. Journal for research in mathematics education, 39(4), 372-400.

Hodgen, J.; \& Askew, M. (junio, 2006). Relationship with/in primary mathematics: identity, emotion and professional development. Proceedings of the British Society for Research into Learning, 26(2), 37-42.

IBM para Windows (versión 24.0) [Programa Informático]. (2016). Armonk, Nueva York: IBM Corp, 2016.

Ma, X., \& Kishor, N. (julio, 1997). Assessing the relationship between attitude toward mathematics and achievement 
in mathematics: A meta-analysis. Journal for research in mathematics education, 28(1), 26-47.

Maaß, J y Schöglmann, W. (2009). Beliefs and attitudes in mathematics education: New research results. Rotterdam: Sense Publishers.

Martín, M. E. (1998). Creencias y prácticas del profesorado de primaria en la enseñanza de las matemáticas (Tesis doctoral, Universidad de La Laguna) . Recuperada de https://es.scribd.com/document/164396808/creencias-ypracticas-del-profesorado-de-primaria-en-la-ensenanzade-las-matematicas

Mcleod, D. B. (1992). Research on affect in mathematics education: A reconceptualization. En J. GROUWS (Ed.), Handbook of research on mathematics teaching and learning,( pp. 575-596). New York: Macmillan Publishing Company.

Mcleod, D. B., Adams, V. M. (2012). Affect and mathematical problem solving: A new perspective. New Yory: SpringerScience y Business Media.

Mellado, V., Blanco, L., Borrachero, A y Cárdenas, J. A. (2012). Las emociones en la enseñanza y el aprendizaje de las ciencias y las matemáticas. España: Grupo de Investigación DEPROFE.

NCTM. (2003). Principios y Estandares para la Educación Matemática, Granada, Servicio de Publicaciones de la SAEM Thales.

Nortes, R y Nortes, A. (agosto, 2014). ¿Tienen ansiedad hacia las matemáticas los futuros matemáticos. Profesorado: Revista de curriculum y formación del profesorado,18(2), 153-170.

Pérez, S y Guillén, G. (2007). Estudio exploratorio sobre creencias y concepciones de profesores de secundaria en relación con la geometría y su enseñanza. En M. Camacho., P. Flores \& P. Bolea (Eds.), Investigación en Educación Matemática XI, La Laguna: SEIEM. Congreso llevado a cabo en La Laguna, Tenerife.

Pezzia, M \& Di Martino, P. (2011): The effect of a teacher education program on affect: the case of Teresa, PFCM.

Philippou, G., \& Christou, C. (febrero, 1998). The effects of a preparatory mathematics program in changing prospective teachers' attitudes towards mathematics. Educational Studies in Mathematics, 35(2), 189-206.

Philippou, G.N\& Christou, C.(2002). Astudy of the mathematics teaching efficacy beliefs of primary teachers". En G.C. Leder., E. Pehkonen \& G. Törner (Eds), Beliefs: a hidden variable in mathematics education?. Congreso llevado a cabo en Dordrecht, Springer.

Picos, A. P., Alonso, S. H., Saez, A. M., Del rincón, T. O. (julio, 2013). Causas y consecuencias de la ansiedad matemática mediante un modelo de ecuaciones estructurales. Enseñanza de las ciencias: revista de investigación y experiencias didácticas, 31(2), 93-111.
Planas, N y Alsina, A. (coords.) (2009). Educación matemática y buenas prácticas. Infantil, primaria,secundaria y educación superior. Barcelona: Editorial GRAÓ.

Real Decreto 126/2014, de 28 de febrero, por el que se establece el currículo básico de la Educación Primaria.

Real Decreto 1105/2014, de 26 de diciembre, por el que se establece el currículo básico de la Educación Secundaria Obligatoria y del Bachillerato.

Rivera, M.F., \& Gómez-Chacón, I.M. (2013). Attitudes towards Mathematics of Teachers in Service of Telesecundaria: an Exploratory Study 1-10. Recuperado de : http://cerme8. metu.edu.tr/wgpapers/WG8/WG8_Fuentes_Rivera.pdf

Ross, J. A., Mcdougall, D., Hogaboam-Gray, A., \& Lesage, A. (julio, 2003). A survey measuring elementary teachers' implementation of standards-based mathematics teaching. Journal for Research in Mathematics Education, 34(4), 344-363.

Sánchez, J., Segovia, I., y Miñän, A. (2011). Exploración de la ansiedad hacia las matemáticas en los futuros maestros de Educación Primaria. Revista de Curriculum y Formación del Profesorado,15(3), 297-312.

Sayers, J. (2007). Primary teachers' attitudes towards and beliefs about mathematics teaching: the collective culture of one English primary school. Working group 12, CERME 5. Congreso llevado a cabo en San Juan, Puerto Rico.

Sweeting, K. (2011). Early year teachers' attitudes towards mathematics (Tesis doctoral, Queensland University of Technology). Recuperada de https://eprints.qut.edu. $\mathrm{au} / 46123 / 1 /$ Kylie_Sweeting_Thesis.pdf

Thiel, O. (marzo, 2010) .Teachers' attitudes towards mathematics in early childhood education. European Early Childhood Education Research Journal, 18(1), 105115.

Uysal, F., \& Dede, Y. (agosto, 2016). Mathematics Anxiety and Beliefs of Turkish Pre-service Elementary Teachers. Eurasia Journal of Mathematics, Science \& Technology Education, 12(8), 2171-2186. 